\title{
SYMBOLIC POWERS OF CERTAIN COVER IDEALS OF GRAPHS
}

\author{
ARVIND KUMAR, RAJIV KUMAR, RAJIB SARKAR, AND S SELVARAJA
}

\begin{abstract}
In this paper, we compute the regularity and Hilbert series of symbolic powers of cover ideal of a graph $G$ when $G$ is either a crown graph or a complete multipartite graph. We also compute the multiplicity of symbolic powers of cover ideals in terms of the number of edges.
\end{abstract}

\section{INTRODUCTION}

Symbolic powers of ideals have been studied intensely over the last two decades. We refer the reader to [4] for a review in this direction. There are many ideals associated to graphs, for example edge ideals and cover ideals. Let $G$ denote a finite simple (no loops, no multiple edges) undirected graph with the vertex set $V(G)=\left\{x_{1}, \ldots, x_{n}\right\}$ and edge set $E(G)$. For a graph $G$, by identifying the vertices with variables in $S=\mathbb{K}\left[x_{1}, \ldots, x_{n}\right]$, where $\mathbb{K}$ is a field, we associate squarefree monomial ideals, edge ideal $I(G)=\left(x_{i} x_{j} \mid\left\{x_{i}, x_{j}\right\} \in E(G)\right)$ and cover ideal $J(G)=\left(\prod_{x \in w} x \mid w\right.$ is a minimal vertex cover of $\left.G\right)$. By [ [6, Proposition 2.7], $I(G)$ and $J(G)$ are dual to each other. Recently, building a dictionary between combinatorial data of graphs and the algebraic properties of corresponding ideals has been done by various authors (cf. [6], [12], [14], [19], [25], 29], [30], [31], 33]). In particular, establishing a relationship between Castelnuovo-Mumford regularity (or simply, regularity) of powers of ideals, Hilbert series of ideals and combinatorial invariants associated with graphs is an active area of research (cf. [1], [9], [21]).

It was proved by Cutkosky, Herzog and Trung [3], and independently Kodiyalam [22], that if $I$ is a homogeneous ideal in $\mathbb{K}\left[x_{1}, \ldots, x_{n}\right]$, then there exist non-negative integers $a, b$ and $s_{0}$ such that $\operatorname{reg}\left(I^{s}\right)=a s+b$ for all $s \geq s_{0}$. While the coefficient $a$ is well-understood (cf. [3], [11], 22], [32]), the free constant $b$ and stablization index $s_{0}=\min \left\{s \mid \operatorname{reg}\left(I^{t}\right)=\right.$ $a t+b$ for $t \geq s\}$ are quite mysterious. In the case of symbolic powers, Minh and Trung [26], ask the following question.

Question 1.1. Let I be a squarefree monomial ideal. Is $\operatorname{reg}\left(I^{(s)}\right)$ a linear function for $s \gg 0$ ?

In [16], Herzog, Hibi and Trung proved that, if $I$ is a monomial ideal, then $\operatorname{reg}\left(I^{(s)}\right)$ is a quasi-linear function for $s \gg 0$. For small dimension, more general results are known in [17] and [18]. It is not known whether the regularity of symbolic powers of squarefree monomial

Key words and phrases. complete multipartite graph, cover ideal, crown graph, Hilbert series, multiplicity, regularity, symbolic power.

AMS Classification 2010: 13D02, 13F20. 
ideals is a linear function or not. In this article, we determine the linear polynomial for the regularity of symbolic powers of certain cover ideals of graphs.

A crown graph $C_{n, n}$ is a graph obtained from $K_{n . n}$ by removing a perfect matching (see definition in Section 2). The Betti numbers of edge ideal and representation number of crown graphs have been looked by several authors [8], [28]. Since crown graph is a bipartite graph, by [7, Corollary 2.6], $J(G)^{s}=J(G)^{(s)}$ for all $s \geq 1$. In [14], Hang and Trung proved that if $G$ is bipartite, then $b \leq|V(G)|-\operatorname{deg}(J(G))-1$ and $s_{0} \leq|V(G)|+2$, where $\operatorname{deg}(J(G))=\max \{|C|: C$ is a minimal vertex cover of $G\}$. In the case of crown graph we obtain that $b=0$ and $s_{0}=1$ (Theorem 3.4).

We then consider complete multipartite graphs. The resolution of powers of cover ideals of complete multipartite graphs and vanishing ideal of the parametrized algebraic toric set associated to complete multipartite graphs have already been studied by several authors [20], [23], [24], [27]. We prove that, if $G$ is complete multipartite with partition $V(G)=$ $V_{1} \cup \cdots \cup V_{k}$, then $\operatorname{reg}\left(J(G)^{(s)}\right)=s \operatorname{deg}(J(G))+p-1$ for all $s \geq 1$, where $p=\min \left\{p_{i}: p_{i}=\left|V_{i}\right|\right\}$ (Theorem 4.6).

The Hilbert function, Hilbert series and Hilbert polynomial are important invariants in commutative algebra and algebraic geometry that measure the growth of the dimension of its homogeneous components. In general, computing the Hilbert series of $S / I$ is a difficult task when $I$ is a monomial ideal [2]. In [9], Goodarzi computed the Hilbert series of squarefree monomial ideals. We compute the Hilbert series of symbolic powers of cover ideals of crown and complete multipartite graphs (Theorem 3.6, Theorem 4.10).

Computing and finding bounds for the multiplicity of homogeneous ideals have been studied by a number of researchers (see [2], 15], 33]). We compute the multiplicity of symbolic powers of cover ideals and edge ideals in terms of combinatorial invariants (Corollary 5.3).

In order to prove our main results, we first show that the minimal monomial generators of symbolic powers of cover ideals have specific order that satisfies some nice properties (Lemma 3.3, Lemma 4.4). Using this ordering and certain exact sequences, we obtain main results.

Our paper is organized as follows. In Section 2, we collect the necessary notion, terminology and some results that are used in rest of the article. The regularity and Hilbert series of symbolic powers of cover ideals of crown and multipartite graphs are discussed in Sections 3 and 4, respectively. The multiplicity of symbolic powers of edge ideals and cover ideals is studied in Section 5 .

\section{Preliminaries}

In this section, we set up basic definitions, notation and some important results which are needed for rest of the paper. 
2.1. Notion from commutative algebra. Let $M=\bigoplus_{k \in \mathbb{N}} M_{k}$ be a finite graded $S$-module. The Hilbert series of $M$, denoted by $H(M, t)$, is defined as $H(M, t):=\sum_{k \in \mathbb{N}} \operatorname{dim}_{\mathbb{K}}\left(M_{k}\right) t^{k}$. By [2, Proposition 4.4.1], there exists a polynomial $h_{M}(t) \in \mathbb{Z}[t]$ such that $H(M, t)=\frac{h_{M}(t)}{(1-t)^{d}}$, where $d$ is the dimension of $M$. The multiplicity of $M$, denoted by $e(M)$, is defined as $e(M)=h_{M}(1)$. The Castelnuovo-Mumford regularity of $M$, denoted by $\operatorname{reg}(M)$, is defined as $\operatorname{reg}(M)=\max \left\{j-i \mid \operatorname{Tor}_{i}^{S}(M, \mathbb{K})_{j} \neq 0\right\}$.

Let $I$ be an ideal in a Noetherian domain $R$. The $s$-th symbolic power of $I$ is defined by $I^{(s)}:=\bigcap_{\mathfrak{p} \in \operatorname{Ass}(R / I)}\left(I^{s} R_{\mathfrak{p}} \cap R\right)$. It follows from [15, Proposition 1.4.4] that if $I$ is a squarefree monomial ideal in $S$, then $s$-th symbolic power of $I$ is $I^{(s)}=\bigcap_{\mathfrak{p} \in \operatorname{Ass}(S / I)} \mathfrak{p}^{s}$.

Remark 2.1. Let $\mathfrak{p}=\left(x_{i_{1}}, \ldots, x_{i_{r}}\right)$. For a monomial $u$ in $S$, set $m_{i}(u)=\max \left\{j: x_{i}^{j} \mid u\right\}$ and $\operatorname{deg}_{\mathfrak{p}}(u)=\sum_{k=1}^{r} m_{i_{k}}(u)$. Let $I$ be a squarefree monomial ideal with $I=\bigcap_{\mathfrak{p} \in \operatorname{Ass}(S / I)} \mathfrak{p}$. Then $u \in I^{(s)}$ if and only if $\operatorname{deg}_{\mathfrak{p}}(u) \geq s$ for all $\mathfrak{p} \in \operatorname{Ass}(S / I)$.

2.2. Notion from combinatorics. Let $G$ be a finite simple graph with the vertex set $V(G)$ and edge set $E(G)$. A subset $X$ of $V(G)$ is called independent if for all $x, y \in X,\{x, y\} \notin$ $E(G)$. A graph $G$ is said to be bipartite if there exist two disjoint independent sets $X$ and $Y$ such that $V(G)=X \cup Y$. A graph $G$ is said to be complete multipartite if $V(G)$ can be partitioned into sets $V_{1}, \ldots, V_{k}$ for some $k \geq 2$ such that $E(G)=\bigcup_{i \neq j}\left\{\{x, y\} \mid x \in V_{i}, y \in V_{j}\right\}$ and it is denoted by $K_{p_{1}, \ldots, p_{k}}$, where $p_{i}=\left|V_{i}\right|$. An $n$-crown graph (or simply a crown graph), denoted by $C_{n, n}$, is a bipartite graph on the vertex set $V(G)=\left\{x_{1}, \ldots, x_{n}, y_{1}, \ldots, y_{n}\right\}$ with edge set $E(G)=\left\{\left\{x_{i}, y_{j}\right\} \mid 1 \leq i, j \leq n, i \neq j\right\}$. A subset $C \subset V(G)$ is a vertex cover of $G$ if for each $e \in E(G), e \cap C \neq \emptyset$. If $C$ is minimal with respect to inclusion, then $C$ is called a minimal vertex cover of $G$.

Example 2.2. Let $G=K_{2,2,1,1}$ and $H=C_{4,4}$ be complete multipartite graph and crown graph on $\left\{x_{1,1}, x_{1,2}, x_{2,1}, x_{2,2}, x_{3,1}, x_{4,1}\right\}$ and $\left\{x_{1}, \ldots, x_{4}, y_{1}, \ldots, y_{4}\right\}$ as given in the figure below.

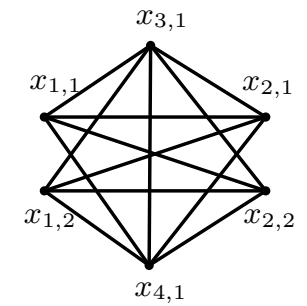

$G$

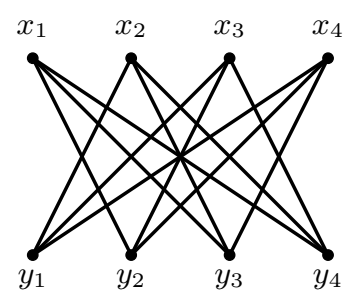

$H$

It can be noted that $\left\{x_{1,1}, x_{1,2}, x_{2,1}, x_{2,2}, x_{3,1}\right\}$ and $\left\{x_{2}, x_{3}, x_{4}, y_{2}, y_{3}, y_{4}\right\}$ are minimal vertex covers of $K_{2,2,1,1}$ and $C_{4,4}$, respectively. 
For any undefined terminology and further basic definitions, we refer the reader to [2], [15].

\section{CROWN GRAPH}

In this section, we study the regularity and Hilbert series of symbolic powers of cover ideals of crown graphs. Throughout this section, $G$ denotes a crown graph.

3.1. Regularity. In this subsection, we obtain the linear function for the regularity of $J(G)^{(s)}$ for all $s \geq 1$. Our result Theorem 3.4 shows that $\operatorname{reg}\left(J(G)^{(s)}\right)$ is a linear function with the stabilization index $s_{0}=1$ and free constant $b=0$. In order to prove this, we first fix certain notation.

Notation 3.1. For $n \geq 3$, let $G=C_{n, n}$ be a graph with $V(G)=\left\{x_{1}, \ldots, x_{n}, y_{1}, \ldots, y_{n}\right\}$. Set

$$
M_{x}=\prod_{i=1}^{n} x_{i}, M_{y}=\prod_{i=1}^{n} y_{i}, M=M_{x} M_{y} \text { and } M_{i}=\frac{M}{x_{i} y_{i}} \text { for } 1 \leq i \leq n .
$$

First, we find the monomial generating set of cover ideal of crown graph.

Lemma 3.2. Let $G=C_{n, n}$ with notation as in 3.1. Then $J(G)=\left(M_{x}, M_{y}, M_{1}, \ldots, M_{n}\right)$. In particular, $\operatorname{deg}(J(G))=2 n-2$.

Proof. Since $J(G)=\bigcap_{i \neq j}\left(x_{i}, y_{j}\right),\left(M_{x}, M_{y}, M_{1}, \ldots, M_{n}\right) \subset J(G)$. Let $u$ be a monomial in $J(G)$. If either $M_{x} \mid u$ or $M_{y} \mid u$, then we are done. Now, we assume that $M_{x} \nmid u$ and $M_{y} \nmid u$. This forces that there exist $i$ and $j$ such that $x_{i} \nmid u$ and $y_{j} \nmid u$. For $k \neq i, J(G) \subset\left(x_{i}, y_{k}\right)$ which forces that $y_{k} \mid u$. This implies that $j=i$, and by symmetry for $k \neq i, x_{k} \mid u$. Therefore, $M_{i} \mid u$ which gives the desired result.

For a monomial $u$, support of $u$, denoted by $\operatorname{supp}(u)$, is defined as $\operatorname{supp}(u)=\left\{x_{i}: x_{i} \mid u\right\}$. The following lemma summarizes some basic properties of $J(G)^{(s)}$.

Lemma 3.3. Let $G=C_{n, n}$ with notation as in 3.1. Then, for $s \geq 2$,

i) $\left(M_{x}\right): M_{y}=\left(M_{x}\right)$.

ii) $\left(M_{x}\right): M_{i}=\left(x_{i}\right)$ and $M_{y}: M_{i}=\left(y_{i}\right)$ for $1 \leq i \leq n$.

iii) $\left(M_{j}\right): M_{i}=\left(x_{i} y_{i}\right)$ for $i \neq j$.

iv) $\left(M_{x}, M_{y}, M_{1}, \ldots, M_{i-1}\right): M_{i}=\left(x_{i}, y_{i}\right)$ for $1 \leq i \leq n$.

v) $J(G)^{s}: M_{x}=J(G)^{s-1}$.

vi) $\left(J(G)^{s}, M_{x}\right): M_{y}=\left(J(G)^{s-1}, M_{x}\right)$.

vii) $\left(J(G)^{s}, M_{x}, M_{y}, M_{1}, \ldots, M_{i-1}\right): M_{i}=\left(x_{i}, y_{i}, M_{i}^{s-1}\right)$ for $1 \leq i \leq n$.

Proof. (i)-(iv) are standard.

(v) The assertion follows from [29, Lemma 3.2].

(vi) Since $\operatorname{supp}\left(M_{x}\right) \cap \operatorname{supp}\left(M_{y}\right)=\emptyset$, by $(\mathrm{v}),\left(J(G)^{s}, M_{x}\right): M_{y}=\left(J(G)^{s-1}, M_{x}\right)$. 
(vii) By (iv), $\left(J(G)^{s}, M_{x}, M_{y}, M_{1}, \ldots, M_{i-1}\right): M_{i} \supset\left(x_{i}, y_{i}, M_{i}^{s-1}\right)$. Let $u$ be a monomial in $\left(J(G)^{s}, M_{x}, M_{y}, M_{1}, \ldots, M_{i-1}\right): M_{i}$. If either $x_{i} \mid u M_{i}$ or $y_{i} \mid u M_{i}$, then $u \in\left(x_{i}, y_{i}, M_{i}^{s-1}\right)$. Suppose $x_{i} \nmid u M_{i}$ and $y_{i} \nmid u M_{i}$. Note that $\left(M_{x}, M_{y}, M_{1}, \ldots, M_{i-1}\right) \subset\left(x_{i}, y_{i}\right)$ and $x_{i} \nmid u M_{i}$ and $y_{i} \nmid u M_{i}$ forces that $u M_{i} \in J(G)^{s}$. Since $G$ is a bipartite graph, by [7, Corollary 2.6], $J(G)^{(s)}=J(G)^{s}$, and hence $J(G)^{s}=\bigcap_{i \neq j}\left(x_{i}, y_{j}\right)^{s}$. For $k \neq i, J(G)^{s} \subset\left(x_{i}, y_{k}\right)^{s}$, and $x_{i} \nmid u M_{i}$ which implies that $y_{k}^{s} \mid u M_{i}$. Note that for $k \neq i, y_{k}^{2} \nmid M_{i}$ which forces that $y_{k}^{s-1} \mid u$. Similarly, we get that $x_{k}^{s-1} \mid u$ for all $k \neq i$. Hence $M_{i}^{s-1} \mid u$.

We now proceed to compute the regularity of powers of $J(G)$.

Theorem 3.4. Let $G=C_{n, n}$. Then for all $s \geq 1$,

$$
\operatorname{reg}\left(J(G)^{s}\right)=s \cdot \operatorname{deg}(J(G)) .
$$

Proof. It follows from [31, Lemma 3.1] that $s \cdot \operatorname{deg}(J(G)) \leq \operatorname{reg}\left(J(G)^{s}\right)$. We need to prove that $\operatorname{reg}\left(J(G)^{s}\right) \leq s \cdot \operatorname{deg}(J(G))$. By [15, Proposition 8.1.10], $\operatorname{reg}(J(G))=\operatorname{pd}(S / I(G))$. If $s=1$, then the result follows from [28, Theorem 4.3]. So, assume that $s \geq 2$. Consider the following short exact sequence:

$$
0 \longrightarrow \frac{S}{J(G)^{s}: M_{x}}(-n) \longrightarrow \frac{S}{J(G)^{s}} \longrightarrow \frac{S}{\left(J(G)^{s}, M_{x}\right)} \longrightarrow 0 .
$$

By Lemma 3.3, $J(G)^{s}: M_{x}=J(G)^{s-1}$. Then, by induction,

$$
\operatorname{reg}\left(J(G)^{s-1}(-n)\right) \leq(s-1) \cdot \operatorname{deg}(J(G))+n \leq s \cdot \operatorname{deg}(J(G)) .
$$

Now, by Equation (3.1), it is sufficient to show that $\left.\operatorname{reg}\left(J(G)^{s}, M_{x}\right)\right) \leq s \cdot \operatorname{deg}(J(G))$.

Claim: $\operatorname{reg}\left(J(G)^{s}, M_{x}\right) \leq s \cdot \operatorname{deg}(J(G))$ for all $s \geq 1$.

Proof of the claim: We proceed by induction on $s$. If $s=1$, then $\left(J(G), M_{x}\right)=J(G)$ and the result follows from [28, Theorem 4.3]. Assume that $s \geq 2$. Set $K=\left(J(G)^{s}, M_{x}\right)$, $K_{1}=\left(K, M_{y}\right)$ and for $2 \leq l \leq n+1, K_{l}=\left(K_{l-1}, M_{l-1}\right)$. Note that $K_{n+1}=J(G)$. Consider the following short exact sequences:

$$
0 \longrightarrow \frac{S}{K: M_{y}}(-n) \longrightarrow \frac{S}{K} \longrightarrow \frac{S}{K_{1}} \longrightarrow 0
$$

for $1 \leq l \leq n$,

$$
0 \longrightarrow \frac{S}{K_{l}: M_{l}}(-(2 n-2)) \longrightarrow \frac{S}{K_{l}} \longrightarrow \frac{S}{K_{l+1}} \longrightarrow 0 .
$$

Using Equations (3.2) and (3.3), we get

$$
\operatorname{reg}(K) \leq \max \left\{\operatorname{reg}\left(K: M_{y}\right)+n, \operatorname{reg}(J(G)), \operatorname{reg}\left(K_{l}: M_{l}\right)+2 n-2 \text { for } 1 \leq l \leq n\right\} .
$$

We now prove that each of regularities appearing on the right hand side of the above inequality is bounded above by $s \cdot \operatorname{deg}(J(G))$. By Lemma 3.3, Theorem 3.4 and [28, Theorem 
4.3], we have

$$
\begin{aligned}
& \operatorname{reg}\left(K: M_{y}\right)=\operatorname{reg}\left(J(G)^{s-1}, M_{x}\right), \operatorname{reg}(J(G))=\operatorname{deg}(J(G)) \\
& \operatorname{reg}\left(K_{l}: M_{l}\right)=\operatorname{reg}\left(x_{l}, y_{l}, M_{l}^{s-1}\right), \text { for all } 1 \leq l \leq n
\end{aligned}
$$

By induction, $\operatorname{reg}\left(K: M_{y}\right) \leq(s-1) \cdot \operatorname{deg}(J(G))$. Since $x_{l}, y_{l}, M_{l}^{s-1}$ is a regular sequence with $\operatorname{deg}\left(x_{l}\right)=\operatorname{deg}\left(y_{l}\right)=1$ and $\operatorname{deg}\left(M_{l}\right)^{s-1}=(s-1) \cdot \operatorname{deg}(J(G)), \operatorname{reg}\left(K_{l}: M_{l}\right)=(s-1) \cdot \operatorname{deg}(J(G))$. Therefore, $\operatorname{reg}\left(J(G)^{s}, M_{x}\right) \leq s \cdot \operatorname{deg}(J(G))$.

3.2. Hilbert series. We compute the Hilbert series of symbolic powers of cover ideals of crown graphs. We begin by computing the Hilbert series of cover ideal.

Theorem 3.5. Let $G=C_{n, n}$ for $n \geq 3$ with notation as in 3.1. Then

$$
H\left(\frac{S}{J(G)}, t\right)=\frac{\sum_{i=0}^{n-1}(i+1) t^{i}+\sum_{i=0}^{n-3}(n-i-1) t^{n+i}-(n-1) t^{2 n-2}}{(1-t)^{2 n-2}} .
$$

Proof. Set $I_{0}=\left(M_{x}, M_{y}\right)$ and $I_{i}=\left(I_{i-1}, M_{i}\right)$ for all $1 \leq i \leq n$. For $1 \leq i \leq n$, consider the exact sequence:

$$
0 \longrightarrow \frac{S}{I_{i-1}: M_{i}}(-(2 n-2)) \longrightarrow \frac{S}{I_{i-1}} \longrightarrow \frac{S}{I_{i}} \longrightarrow 0 .
$$

We have $H\left(\frac{S}{J(G)}, t\right)=H\left(\frac{S}{I_{0}}, t\right)-t^{2 n-2} \sum_{i=1}^{n} H\left(\frac{S}{I_{i-1}: M_{i}}, t\right)$. Since $M_{x}, M_{y}$ is a regular sequence on $S$ of degree $n$, we get $H\left(\frac{S}{I_{0}}, t\right)=\frac{\left(1-t^{n}\right)^{2}}{(1-t)^{2 n}}$. By Lemma 3.3, $I_{i-1}: M_{i}=\left(x_{i}, y_{i}\right)$ for any $1 \leq i \leq n$ which implies that $H\left(\frac{S}{I_{i-1}: M_{i}}, t\right)=\frac{1}{(1-t)^{2 n-2}}$. Hence

$$
H\left(\frac{S}{J(G)}, t\right)=\frac{\sum_{i=0}^{n-1}(i+1) t^{i}+\sum_{i=0}^{n-3}(n-i-1) t^{n+i}-(n-1) t^{2 n-2}}{(1-t)^{2 n-2}} .
$$

We end this section by proving the following main result.

Theorem 3.6. Let $G=C_{n, n}$ for $n \geq 3$ with notation as in 3.1. Then for all $s \geq 1$, $H\left(\frac{S}{J(G)^{s}}, t\right)$

$$
=\frac{\sum_{i=0}^{n s-1}(i+1) t^{i}+\sum_{i=0}^{n-3}(n-i-1) s t^{n s+i}-(n-1) s t^{n s+n-2}-\sum_{i=0}^{s-2}(i+1) n t^{s(2 n-2)-i(n-2)}}{(1-t)^{2 n-2}} .
$$


Proof. We proceed by induction on $s$. By Theorem 3.5, the result is true for $s=1$. Assume that $s \geq 2$. Using Lemma 3.3 (v) and exact sequence (3.1), we get

$$
H\left(\frac{S}{J(G)^{s}}, t\right)=t^{n} H\left(\frac{S}{J(G)^{s-1}}, t\right)+H\left(\frac{S}{\left(J(G)^{s}, M_{x}\right)}, t\right) .
$$

Claim: For all $s \geq 1, H\left(\frac{S}{\left(J(G)^{s}, M_{x}\right)}, t\right)$

$$
=\frac{\sum_{i=0}^{n-1}(i+1) t^{i}+n \sum_{i=n}^{n s-1} t^{i}+\sum_{i=1}^{n-2}(n-i) t^{n s+i-1}-(n-1) t^{n s+n-2}-n \sum_{i=0}^{s-2} t^{s(2 n-2)-i(n-2)}}{(1-t)^{2 n-2}} .
$$

Now, it is enough to prove the above claim as the desired result follows from induction argument, claim and Equation (3.4).

Proof of the claim: For $s=1$ the result follows from Theorem 3.5 and the fact that $\left(J(G), M_{x}\right)=J(G)$. Assume that $s \geq 2$. Using Equations (3.2) and (3.3), we get

$$
H\left(\frac{S}{K}, t\right)=t^{n} H\left(\frac{S}{K: M_{y}}, t\right)+\sum_{i=1}^{m} t^{2 n-2} H\left(\frac{S}{K_{i}: M_{i}}, t\right)+H\left(\frac{S}{J(G)}, t\right) .
$$

By Lemma 3.3(vi) and (vii), $K: M_{y}=\left(J(G)^{s-1}, M_{x}\right)$ and $K_{i}: M_{i}=\left(x_{i}, y_{i}, M_{i}^{s-1}\right)$. Since $x_{i}, y_{i}, M_{i}^{s-1}$ is a regular sequence with $\operatorname{deg}\left(M_{i}\right)=2 n-2$, we get that

$$
H\left(\frac{S}{K_{i}: M_{i}}, t\right)=\frac{\left(1-t^{(s-1)(2 n-2)}\right)}{(1-t)^{2 n-2}} .
$$

Now the claim follows from Equation (3.5), Theorem 3.5 and induction.

\section{Complete multipartite Graph}

In this section, we study the regularity and Hilbert series of symbolic powers of cover ideals of complete multipartite graphs. Throughout this section, $G$ denotes a complete multipartite graph.

4.1. Regularity. We determine the regularity of symbolic powers of cover ideals of complete multipartite graphs. In order to compute the regularity of $J(G)^{(s)}$, we first find the generators of $J(G)$ and its symbolic powers. We begin by fixing some notation which are used for the rest of this section.

Notation 4.1. Let $G=K_{p_{1}, \ldots, p_{k}}$ be a complete multipartite graph with the vertex set

$$
V(G)=\bigcup_{i=1}^{k}\left\{x_{i j}: 1 \leq j \leq p_{i}\right\}, p_{1} \geq p_{2} \geq \cdots \geq p_{k} \geq 1 \text { and } k \geq 2 .
$$

Set

$n=p_{1}+p_{2}+\cdots+p_{k}, M_{i}=\prod_{j=1}^{p_{i}} x_{i j}$ for $1 \leq i \leq k, M=\prod_{i=1}^{k} M_{i}$ and $N_{i}=\frac{M}{M_{i}}$ for $1 \leq i \leq k$. 
The following lemma describes the minimal monomial generating set of $J(G)$.

Lemma 4.2. Let $G=K_{p_{1}, \ldots, p_{k}}$ with the notation as in 4.1. Then $J(G)=\left(N_{i} \mid 1 \leq i \leq k\right)$. In particular, $\operatorname{deg}(J(G))=n-p_{k}$.

Proof. Note that $\operatorname{supp}\left(N_{i}\right)$ is a vertex cover of $G$ which implies that $\left(N_{i} \mid 1 \leq i \leq k\right) \subset J(G)$. Let $u$ be a monomial in $J(G)$. If for all $i, j, x_{i, j} \mid u$, then $u \in\left(N_{i} \mid 1 \leq i \leq k\right)$. Now, without loss of generality, assume that $x_{1,1} \nmid u$. Since for all $i \neq 1$ and $j, J(G) \subset\left(x_{1,1}, x_{i, j}\right)$, we get $x_{i, j} \mid u$, which further implies that $N_{1} \mid u$. This completes the proof.

If $k=2$, then by [7, Corollary 2.6], $J(G)^{s}=J(G)^{(s)}$ for all $s \geq 1$. If $k \geq 3$, then $G$ is non-bipartite graph and every vertex in $G$ is adjacent to every odd cycle in $G$. Therefore, by [5, Theorem 4.9 and Remark 4.10], $J(G)^{(s)}=M J(G)^{(s-2)}+J(G)^{s}$. Now, we further reduce the above expression.

Lemma 4.3. Let $G=K_{p_{1}, \ldots, p_{k}}$ with the notation as in 4.1. Then

$$
J(G)^{(s)}=M J(G)^{(s-2)}+\left(N_{j}^{s} \mid j \in[k]\right) .
$$

Proof. For $e \in E(G), \mathfrak{p}_{e}$ denote an ideal generated by end points of $e$. Let $u$ be a monomial $J(G)^{(s)}$. By Remark 2.1, for every $e \in E(G)$, we have $\operatorname{deg}_{\mathfrak{p}_{e}}(u) \geq s$. Note that $\operatorname{deg}_{\mathfrak{p}_{e}}(M)=2$. If $u=M v$, then $\operatorname{deg}_{\mathfrak{p}_{e}}(v) \geq s-2$ which implies that $v \in J(G)^{(s-2)}$.

Suppose $M \nmid u$. Then there exists $x_{i, j}$ such that $x_{i, j}$ does not divide $u$. Without loss of generality, we may assume that $x_{1,1} \nmid u$. Since $x_{1,1}$ is adjacent to $x_{i, j}$ for $i \geq 2$ and for all $j$, by Remark 2.1, $N_{1}^{s}$ divides $u$. Thus, we have $J(G)^{(s)} \subset M J(G)^{(s-2)}+\left(N_{j}^{s}: j \in[k]\right)$. Clearly, $\left(N_{j}^{s}: j \in[k]\right) \subset J(G)^{(s)}$. It follows from Remark 2.1 that $M J(G)^{(s-2)} \subset J(G)^{(s)}$ which completes the proof.

For a monomial ideal $I=\left(m_{1}, \ldots, m_{r}\right)$, let $I^{[s]}$ denote an ideal generated by $m_{1}^{s}, \ldots, m_{r}^{s}$. The following lemma plays a crucial role to compute the regularity of $J(G)^{(s)}$.

Lemma 4.4. Let $G=K_{p_{1}, \ldots, p_{k}}$ with the notation as in 4.1. Then

i) $J(G)^{(s)}: M=J(G)^{(s-2)}$ for $s \geq 2$.

ii) $\left(J(G)^{(s)}, M\right)=\left(J(G)^{[s]}, M\right)$ for $s \geq 1$.

iii) $\left(N_{1}^{s}, \ldots, N_{i-1}^{s}\right): N_{i}^{s}=\left(M_{i}^{s}\right)$ for $2 \leq i \leq k$.

iv) $J(G)^{[s]}: M=J(G)^{[s-1]}$ for $s \geq 2$.

Proof. (i) and (ii) follow from [30, Lemma 3.4] and Lemma 4.3, respectively.

(iii) Clearly $\left(M_{i}^{s}\right) \subset\left(N_{1}^{s}, \ldots, N_{i-1}^{s}\right): N_{i}^{s}$. Let $u$ be a monomial in $\left(N_{1}^{s}, \ldots, N_{i-1}^{s}\right): N_{i}^{s}$. This forces that for some $1 \leq j<i, N_{j}^{s} \mid u N_{i}^{s}$ which implies that $M_{i}^{s} \mid u$.

(iv) If $u \in J(G)^{[s]}: M$, then there exists $i$ such that $N_{i}^{s} \mid u M$ and so $N_{i}^{s-1} \mid u$. On the other side, since $N_{i} \mid M$ for all $i$, we get $N_{i}^{s} \mid M N_{i}^{s-1}$. 
For fixed $s \geq 2$ and $j \in[k]$, we associate an ideal $I_{s, j}=\left(M, N_{1}^{s}, \ldots, N_{j}^{s}\right)$. Now, we compute the regularity of $I_{s, j}$ in terms of $s$ and $p_{j}$, which helps to compute the regularity of $J(G)^{(s)}$.

Lemma 4.5. For fixed $s \geq 2$ and $j \in[k], \operatorname{reg}\left(I_{s, j}\right)=s\left(n-p_{j}\right)+p_{j}-1$.

Proof. We prove the assertion by induction on $j$. Suppose $j=1$, consider the exact sequence

$$
0 \longrightarrow \frac{S}{M: N_{1}^{s}}\left(-s\left(n-p_{1}\right)\right) \longrightarrow \frac{S}{M} \longrightarrow \frac{S}{\left(M, N_{1}^{s}\right)} \longrightarrow 0 .
$$

Note that $\operatorname{reg}(M)=n$ and $\left(M: N_{1}^{s}\right)=\left(M_{1}\right)$. Therefore,

$$
\operatorname{reg}\left(\left(M: N_{1}^{s}\right)\left(-s\left(n-p_{1}\right)\right)\right)=p_{1}+s\left(n-p_{1}\right)
$$

Since $s \geq 2$, it follows from [13, Lemma $1.2(\mathrm{v})]$ that $\operatorname{reg}\left(M, N_{1}^{s}\right)=s\left(n-p_{1}\right)+p_{1}-1$. Consider the exact sequence

$$
0 \longrightarrow \frac{S}{I_{s, j-1}: N_{j}^{s}}\left(-s\left(n-p_{j}\right)\right) \longrightarrow \frac{S}{I_{s, j-1}} \longrightarrow \frac{S}{I_{s, j}} \longrightarrow 0 .
$$

Note that $\left(I_{s, j-1}: N_{j}^{s}\right)=\left(M_{j}\right)$, and hence reg $\left(\left(I_{s, j-1}: N_{j}^{s}\right)\left(-s\left(n-p_{j}\right)\right)\right)=s\left(n-p_{j}\right)+p_{j}$. By induction, $\operatorname{reg}\left(I_{s, j-1}\right)=s\left(n-p_{j-1}\right)+p_{j-1}-1$. Hence by [13, Lemma $1.2(\mathrm{v})$ ], we get $\operatorname{reg}\left(I_{s, j}\right)=s\left(n-p_{j}\right)+p_{j}-1$.

We now compute the regularity of $J(G)^{(s)}$. Since complete multipartite graph is a matroid, there is another way to compute the regularity of $J(G)^{(s)}$, see [26, Theorem 4.5]. We have provided here an elementary proof so that the result is accessible to readers who are not familiar with matroid.

Theorem 4.6. Let $G=K_{p_{1}, \ldots, p_{k}}$ with the notation as in 4.1. Then for all $s \geq 1$,

$$
\operatorname{reg}\left(J(G)^{(s)}\right)=s \cdot \operatorname{deg}(J(G))+p_{k}-1 .
$$

Proof. We prove the result by induction on $s$. If $s=1$, then the result follows from [19, Theorem 5.3.8]. Assume that $s>1$. Consider the following exact sequence:

$$
0 \longrightarrow \frac{S}{J(G)^{(s)}: M}(-n) \longrightarrow \frac{S}{J(G)^{(s)}} \longrightarrow \frac{S}{\left(J(G)^{(s)}, M\right)} \longrightarrow 0 .
$$

By Lemma 4.4(i), $J(G)^{(s)}: M=J(G)^{(s-2)}$ and by induction

$$
\operatorname{reg}\left(\left(J(G)^{(s)}: M\right)(-n)\right)=(s-2) \cdot \operatorname{deg}(J(G))+n+p_{k}-1 .
$$

It follows from Lemma 4.3 that $\left(J(G)^{(s)}, M\right)=I_{s, k}$. Now, by Lemma 4.5, we get

$$
\operatorname{reg}\left(J(G)^{(s)}, M\right)=s \cdot \operatorname{deg}(J(G))+p_{k}-1 .
$$

Hence the assertion follows [13, Lemma 1.2].

It follow from Theorem 4.6 that if $p_{k}=1$, then the free constant $b=0$. 
Corollary 4.7. Let $G$ be a complete graph on $n$ vertices. Then, for all $s \geq 1$,

$$
\operatorname{reg}\left(J(G)^{(s)}\right)=s \cdot \operatorname{deg}(J(G))=s(n-1) .
$$

4.2. Hilbert series. In this subsection, we compute the Hilbert series of symbolic powers of $J(G)$ in terms of number of vertices and cardinality of partition. To accomplish this, we first study the Hilbert series of $\frac{S}{J(G)^{[s]}}$ for all $s \geq 1$.

Proposition 4.8. Let $G=K_{p_{1}, \ldots, p_{k}}$ with the notation as in 4.1. Then, for all $s \geq 1$,

$$
H\left(\frac{S}{J(G)^{[s]}}, t\right)=\frac{1-\sum_{i=1}^{k} t^{s\left(n-p_{i}\right)}+(k-1) t^{s n}}{(1-t)^{n}} .
$$

Proof. By Lemma 4.4(iii), $\left(N_{1}^{s}, \ldots, N_{i-1}^{s}\right): N_{i}^{s}=\left(M_{i}^{s}\right)$ for all $2 \leq i \leq k$. Now, for $2 \leq i \leq k$ consider the exact sequences:

$$
0 \longrightarrow \frac{S}{\left(M_{i}^{s}\right)}\left(-s\left(n-p_{i}\right)\right) \longrightarrow \frac{S}{\left(N_{1}^{s}, \ldots, N_{i-1}^{s}\right)} \longrightarrow \frac{S}{\left(N_{1}^{s}, \ldots, N_{i}^{s}\right)} \longrightarrow 0 .
$$

We know that $H\left(\frac{S}{\left(M_{i}^{s}\right)}, t\right)=\frac{1-t^{s p_{i}}}{(1-t)^{n}}$ for all $2 \leq i \leq k$. Therefore, by applying successively the above short exact sequences, we get

$$
\begin{aligned}
H\left(\frac{S}{J(G)^{[s]}}, t\right) & =H\left(\frac{S}{\left(N_{1}^{s}\right)}, t\right)-\sum_{i=2}^{k} t^{s\left(n-p_{i}\right)} H\left(\frac{S}{\left(M_{i}^{s}\right)}, t\right) \\
& =\frac{1-t^{s\left(n-p_{1}\right)}}{(1-t)^{n}}-\sum_{i=2}^{k}\left(\frac{t^{s\left(n-p_{i}\right)}-t^{s n}}{(1-t)^{n}}\right) \\
& =\frac{1-\sum_{i=1}^{k} t^{s\left(n-p_{i}\right)}+(k-1) t^{s n}}{(1-t)^{n}} .
\end{aligned}
$$

To obtain the Hilbert series of $\frac{S}{J(G)^{(s)}}$, we need the following lemma:

Lemma 4.9. Let $G=K_{p_{1}, \ldots, p_{k}}$ with the notation as in 4.1. Then for all $s \geq 1$,

$$
H\left(\frac{S}{\left(J(G)^{[s]}, M\right)}, t\right)=\frac{1-t^{n}-\sum_{i=1}^{k} t^{s\left(n-p_{i}\right)}+\sum_{i=1}^{k} t^{s\left(n-p_{i}\right)+p_{i}}}{(1-t)^{n}} .
$$

Proof. Consider the short exact sequence:

$$
0 \longrightarrow \frac{S}{J(G)^{[s]}: M}(-n) \longrightarrow \frac{S}{J(G)^{[s]}} \longrightarrow \frac{S}{\left(J(G)^{[s]}, M\right)} \longrightarrow 0 .
$$


By Lemma 4.4(iv), $J(G)^{[s]}: M=J(G)^{[s-1]}$. Therefore

$$
H\left(\frac{S}{\left(J(G)^{[s]}, M\right)}, t\right)=H\left(\frac{S}{J(G)^{[s]}}, t\right)-t^{n} H\left(\frac{S}{J(G)^{[s-1]}}, t\right) .
$$

Using Proposition 4.8, we get the result.

We are now ready to establish the Hilbert series of $\frac{S}{J(G)^{(s)}}$ for all $s \geq 1$.

Theorem 4.10. Let $G=K_{p_{1}, \ldots, p_{k}}$ with the notation as in 4.1. Then $H\left(\frac{S}{J(G)^{(s)}}, t\right)$ is

$$
\left\{\begin{array}{cc}
\frac{1-t^{r n}+\sum_{j=0}^{r-1} \sum_{i=1}^{k}\left(t^{p_{i}}-1\right) t^{(s-j)\left(n-p_{i}\right)+j p_{i}}}{(1-t)^{n}}, & \text { if } s=2 r, r \geq 1 \\
\frac{1+(k-1) t^{(r+1) n}-\sum_{i=1}^{k} t^{\left(n-p_{i}\right)+r n}+\sum_{j=0}^{r-1} \sum_{i=1}^{k}\left(t^{p_{i}}-1\right) t^{(s-j)\left(n-p_{i}\right)+j p_{i}}}{(1-t)^{n}} & \text { if } s=2 r+1, r \geq 0 .
\end{array}\right.
$$

Proof. It follows from Lemma 4.4(i) and (ii) that for $s \geq 2, J(G)^{(s)}: M=J(G)^{(s-2)}$ and $\left(J(G)^{(s)}, M\right)=\left(J(G)^{[s]}, M\right)$. Using Equation (4.1), we get

$$
H\left(\frac{S}{J(G)^{(s)}}, t\right)=t^{n} H\left(\frac{S}{J(G)^{(s-2)}}, t\right)+H\left(\frac{S}{\left(J(G)^{[s]}, M\right)}, t\right) .
$$

Suppose $s=2 r$. We prove this by induction on $r$. If $r=1$, then, by Lemma 4.3 , $J(G)^{(2)}=\left(J(G)^{[2]}, M\right)$. Now the result follows from Lemma 4.9, Assume that $r \geq 2$. Now by induction and Lemma 4.9, we get the assertion.

Suppose $s=2 r+1$. By Lemma 4.8, the result holds for $r=0$. Now assume that $r \geq 1$. The assertion follows from induction, Lemma 4.9 and Equation (4.3).

\section{Multiplicity}

In this section, we study the multiplicity of symbolic powers of cover ideals and edge ideals. The following lemma is probably well-known. We include it for the sake of completeness.

Lemma 5.1. Let I be minimally generated by h linear forms. Then $e\left(\frac{S}{I^{s}}\right)=\left(\begin{array}{c}s+h-1 \\ h\end{array}\right)$.

Proof. Let $x \in \mathfrak{m} \backslash I$ be a generator of $\mathfrak{m}$, where $\mathfrak{m}=\left(x_{1}, \ldots, x_{n}\right)$ is the unique homogeneous maximal ideal in $S$. Then $x$ is a regular element on $I$, and so on $I^{s}$. This gives $H\left(\frac{S}{\left(I^{s}, x\right)}, t\right)=(1-t) H\left(\frac{S}{I^{s}}, t\right)$, and hence $e\left(\frac{S}{\left(I^{s}, x\right)}\right)=e\left(\frac{S}{I^{s}}\right)$. Therefore, without loss of generality, we may assume that $I=\mathfrak{m}$. Thus, we get $e\left(\frac{S}{I^{s}}\right)=\operatorname{dim}_{\mathbb{K}}\left(\frac{S}{I^{s}}\right)$. 
Observation 5.2. Let $I$ be a squarefree monomial ideal in $S$. Since the minimal associated primes of squarefree monomial ideals are generated by subsets of variables, by [2, Corollary 4.7.8] and Lemma 5.1, we have

$$
e\left(\frac{S}{I^{(s)}}\right)=\left(\begin{array}{c}
h+s-1 \\
h
\end{array}\right)|\operatorname{Minh}(I)|,
$$

where $\operatorname{Minh}(I)=\{\mathfrak{p} \in \operatorname{Ass}(S / I): \operatorname{ht}(\mathfrak{p})=\operatorname{ht}(I)\}$ and $h=\operatorname{ht}(I)$

As a consequence of Observation 5.2, we obtain the multiplicity of symbolic powers of edge ideals and cover ideals in terms of combinatorial invariants.

Corollary 5.3. Let $G$ be a graph and $h$ be the size of the smallest vertex cover of $G$. Then for all $s \geq 1$,

i) $e\left(\frac{S}{I(G)^{(s)}}\right)=\left(\begin{array}{c}h+s-1 \\ h\end{array}\right) \mathcal{V}(G)$, where $\mathcal{V}(G)$ is the number of minimal vertex covers of $G$ of minimal size.

ii) $e\left(\frac{S}{J(G)^{(s)}}\right)=\left(\begin{array}{c}s+1 \\ 2\end{array}\right)|E(G)|$.

Acknowledgement: We would like to thank A. V. Jayanthan and J. K. Verma for clarifications on several doubts. The computational commutative algebra package Macaulay 2 [10] was heavily used to compute several examples. The first named author is partially supported by NBHM, India. The third named author is partially supported by UGC, India. The last named author is partially supported by the Institute of Mathematical Sciences, Chennai and National Postdoctoral Fellowship (PDF/2019/002800) by Sciences and Engineering Research Board, India. We also thank the referee for carefully reading the manuscript and making several suggestions that improved the exposition.

\section{REFERENCES}

[1] S. Beyarslan, H. T. Hà, and T. N. Trung. Regularity of powers of forests and cycles. J. Algebraic Combin., 42(4):1077-1095, 2015.

[2] W. Bruns and J. Herzog. Cohen-Macaulay rings, volume 39 of Cambridge Studies in Advanced Mathematics. Cambridge University Press, Cambridge, 1993.

[3] S. D. Cutkosky, J. Herzog, and N. V. Trung. Asymptotic behaviour of the Castelnuovo-Mumford regularity. Compositio Math., 118(3):243-261, 1999.

[4] H. Dao, A. De Stefani, E. Grifo, C. Huneke, and L. Núñez Betancourt. Symbolic powers of ideals. In Singularities and foliations. geometry, topology and applications, volume 222 of Springer Proc. Math. Stat., pages 387-432. Springer, Cham, 2018.

[5] B. Drabkin and L. Guerrieri. Asymptotic invariants of ideals with Noetherian symbolic Rees algebra and applications to cover ideals. J. Pure Appl. Algebra, 224(1):300-319, 2020.

[6] C. A. Francisco, H. T. Hà, and J. Mermin. Powers of square-free monomial ideals and combinatorics. In Commutative algebra, pages 373-392. Springer, New York, 2013. 
[7] I. Gitler, E. Reyes, and R. H. Villarreal. Blowup algebras of ideals of vertex covers of bipartite graphs. In Algebraic structures and their representations, volume 376 of Contemp. Math., pages 273-279. Amer. Math. Soc., Providence, RI, 2005.

[8] M. Glen, S. Kitaev, and A. Pyatkin. On the representation number of a crown graph. Discrete Appl. Math., 244:89-93, 2018.

[9] A. Goodarzi. On the Hilbert series of monomial ideals. J. Combin. Theory Ser. A, 120(2):315-317, 2013.

[10] D. R. Grayson and M. E. Stillman. Macaulay2, a software system for research in algebraic geometry. Available at http://www.math.uiuc.edu/Macaulay2/.

[11] Y. Gu. Regularity of powers of edge ideals of some graphs. Acta Math. Vietnam., 42(3):445-454, 2017.

[12] H. T. Hà and N. V. Trung. Membership criteria and containments of powers of monomial ideals. Acta Math. Vietnam., 44(1):117-139, 2019.

[13] H. T. Hà, N. V. Trung, and T. N. Trung. Depth and regularity of powers of sums of ideals. Math. Z., 282(3-4):819-838, 2016.

[14] N. T. Hang and T. N. Trung. Regularity of powers of cover ideals of unimodular hypergraphs. J. Algebra, 513:159-176, 2018.

[15] J. Herzog and T. Hibi. Monomial ideals, volume 260 of Graduate Texts in Mathematics. Springer-Verlag London, Ltd., London, 2011.

[16] J. Herzog, T. Hibi, and N. V. Trung. Symbolic powers of monomial ideals and vertex cover algebras. Adv. Math., 210(1):304-322, 2007.

[17] J. Herzog, L. T. Hoa, and N. V. Trung. Asymptotic linear bounds for the Castelnuovo-Mumford regularity. Trans. Amer. Math. Soc., 354(5):1793-1809, 2002.

[18] L. T. Hoa and T. N. Trung. Partial Castelnuovo-Mumford regularities of sums and intersections of powers of monomial ideals. Math. Proc. Cambridge Philos. Soc., 149(2):229-246, 2010.

[19] S. Jacques. Betti numbers of graph ideals. PhD thesis, University of Sheffield, 2004.

[20] A. V. Jayanthan and N. Kumar. Syzygies, Betti Numbers, and regularity of cover ideals of certain multipartite graphs. Mathematics, 869(7), 2019.

[21] A. V. Jayanthan, N. Narayanan, and S. Selvaraja. Regularity of powers of bipartite graphs. J. Algebraic Combin., 47(1):17-38, 2018.

[22] V. Kodiyalam. Asymptotic behaviour of Castelnuovo-Mumford regularity. Proc. Amer. Math. Soc., 128(2):407-411, 2000.

[23] A. Kumar and R. Kumar. Regularity, Rees algebra and Betti numbers of certain cover ideals. In Prepartion.

[24] R. Kumar and A. Kumar. Certain classes of Cohen-Macaulay multipartite graphs. Comm. Algebra, 47(5):1930-1938, 2019.

[25] J. Martínez-Bernal, S. Morey, R. H. Villarreal, and C. E. Vivares. Depth and regularity of monomial ideals via polarization and combinatorial optimization. Acta Math. Vietnam., 44(1):243-268, 2019.

[26] N. C. Minh and T. N. Trung. Regularity of symbolic powers and arboricity of matroids. Forum Math., 31(2):465-477, 2019.

[27] J. Neves and M. Vaz Pinto. Vanishing ideals over complete multipartite graphs. J. Pure Appl. Algebra, 218(6):1084-1094, 2014.

[28] S. A. Rather and P. Singh. On betti numbers of edge ideals of crown graphs. Beiträge zur Algebra und Geometrie / Contributions to Algebra and Geometry, 2018.

[29] S. A. Seyed Fakhari. Depth, Stanley depth, and regularity of ideals associated to graphs. Arch. Math. (Basel), 107(5):461-471, 2016. 
[30] S. A. Seyed Fakhari. Depth and Stanley depth of symbolic powers of cover ideals of graphs. J. Algebra, 492:402-413, 2017.

[31] S. A. Seyed Fakhari. Regularity of symbolic powers of cover ideals of graphs. Collect. Math., 70(2):187$195,2019$.

[32] N. V. Trung and H.-J. Wang. On the asymptotic linearity of Castelnuovo-Mumford regularity. J. Pure Appl. Algebra, 201(1-3):42-48, 2005.

[33] R. H. Villarreal. Monomial algebras. Monographs and Research Notes in Mathematics. CRC Press, Boca Raton, FL, second edition, 2015.

Email address: arvkumar11@gmail.com

Department of Mathematics, Indian Institute of Technology Madras, Chennai, india 600036

Email address: gargrajiv00@gmail.com

Department of Mathematics The LnM institute OF Information Technology Jaipur, INDIA-302031

Email address: rajib.sarkar63@gmail.com

Department of Mathematics, Indian Institute of Technology Madras, Chennai, indiA 600036

Email address: selva.y2s@gmail.com, selvaraja@imsc.res.in

The Institute of Mathematical Sciences, Cit campus, Taramani, Chennai, india - 600113 